\title{
Cognitive and Affective Components of Life Events: Their Relations and Effects on Well-Being 1
}

\author{
Amiram Vinokur ${ }^{2}$ and Robert D. Caplan \\ Institute for Social Research, The University of Michigan
}

A model of how five key facets of life events are related to one another and influence mental health and functioning was examined. The facets are the experience of control over, anticipation of, pleasantness from, stress generated by, and adjustment to life events. Multivariate analyses of data from 420 adult male respondents yielded results that were largely consistent with the hypothesized model. The results suggested that control over and anticipation of life events influenced the perceived stress produced by the events and the ability to adjust to them. In turn, it appeared that such stress and adjustment influenced mental health and functioning. The effects of control and anticipation on mental health and functioning were indirect only. Contrary to our hypothesis, control seemed to heighten perceived stress; but control, as well as anticipation, appeared to increase the ability to adjust. Whereas previous studies found no effect of desirable events on well-being, this study found that pleasant events had a beneficial effect. The discussion examines the implications of the findings for future research.

In the nearly 20 years since Holmes and Rahe (1967) published the Social Readjustment Rating Scale, there has been a voluminous literature on how life events influence health, adjustment, and well-being. This literature has produced considerable evidence that life events, particularly negative ones, are associated with poor physical and mental health (for a comprehensive review see Thoits, 1983).

\footnotetext{
${ }^{1}$ The work reported in this paper was supported by a National Institute of Mental Health Grant MH34586.

${ }^{2}$ All correspondence should be sent to Amiram Vinokur, Institute for Social Research, The University of Michigan, Ann Arbor, Michigan 48106.
} 
This study deals with three questions that arise from this literature. First, why is the evidence for a link between the amount of control over and anticipation of life events and mental health so inconsistent (Thoits, 1983)? Second, how do multiple events have their deleterious effect on well-being? Are their effects additive as assumed by the current practice of summing life change (or stress rating) scores, or alternatively, do they interact with earlier events exacerbating the effects of later ones?

Third, what constitutes a negative event? There is considerable evidence that negative events have particularly deleterious effects on mental health (e.g., Fontana, Hughes, Marcus, \& Dowds, 1979; Gersten, Langner, Eisenberg, \& Orzek, 1974; Mueller, Edwards, \& Yarvis, 1977; Ross \& Mirowsky, 1979; Vinokur \& Selzer, 1975). Despite the demonstrated importance of distinguishing between negative and positive events, there is still an absence of information on those basic properties of negative events that determine their adverse effects on well-being. In a similar vein, there is the question of why positive events are not found to have beneficial effects on well-being (or preventive effects on poor mental health), whereas negative events almost always appear to adversely influence mental health and well-being.

Each of the three issues mentioned above are elaborated on briefly. Then these issues are integrated into an interrelated set of hypotheses or a theory regarding the links among critical properties of life events and how they influence well-being. First, however, we define control, stress, adjustment, and well-being because they are prominent in the ensuing set of hypotheses.

In this study control refers to the person's ability to regulate the onset, intensity, and cessation of the life event (Cohen, 1980). The stress of a life event refers to a state of imbalance, or a misfit, between the demands posed by the event and the response capacity of the individual to meet the demands (e.g., French, Rodgers, \& Cobb, 1974; McGrath, 1970). Conversely, adjustment refers to the extent to which the person has reachieved person-environment fit in recovering from the stress or demands posed by the event. Finally, well-being is conceived of in two complementary ways: First, it is conceived of in terms of the person's emotional and role functioning, that is, the ability to handle interpersonal role relationships, problems and associated emotions. Second, it is conceived of as a low level of symtomatology associated with poor mental health.

\section{Why Don't Control Over and Anticipation of Life Events Influence Mental Health?}

A considerable body of research demonstrates that lack of control over stressors and inability to predict their onset interferes with people's abilities 
to overcome many types of adversity (see review by Cohen, 1980; Janis, 1962; for exceptions see Folkman, 1984, pp. 845-846). Yet, lack of perceived control and of predictability do not appear to intensify the effects of life events on well-being (Thoits, 1983). This study presents and tests a theoretical model that suggests that control and anticipation do have an effect on mental health but that this effect is an indirect one holding only insofar as lack of control and of anticipation increase the severity of the event as a stressor and reduce the ability to adjust to it.

\section{How Do Multiple Life Events Influence Well-Being?}

One process may be a buildup or accumulation of change produced by all events, as was proposed originally by Holmes and Rahe, (1967), or by negative, unpleasant events, as is now proposed. It is also possible that the highest or the average or the most recent level of stress attained is the key determinant of ill-being. ${ }^{3}$

It is further possible that earlier events exacerbate the effects of later ones; as the number of events increases, the person's resistance or ability to withstand such stressors drops. If so, then the number of events may increase the mean level of stress per event. This study explores these issues by examining the relation between the number of events and the resultant mean level of stress per event.

\section{What Constitutes a Negative Event?}

Both cognitive and affective factors may determine the negative aspects of an event. Regarding the role of cognitions, previous research suggests that when respondents rate events in terms of being desirable (or good) or undesirable (or bad) they may be focusing on the meaning of the events' outcomes. For example, one study found that half of the men who lost their wives during the preceding year rated the event as desirable (Vinokur \& Selzer, 1975). Many of these men explained to their interviewers that their wife had suffered from a debilitating and painful terminal illness. There was no hope for improvement, and consequently death was viewed as producing a desirable outcome.

Rather than the cognitive meaning of the event's outcome, the quality of the affective reaction that accompanies the event may be the most important facet of how the event is experienced. Is it experienced as a pleasant

${ }^{3}$ One might also examine the most recent event as the key determinant; this study does not have data on the sequencing among a series of recent life events to allow a test of that model. 
or an unpleasant event? The present study examined how this emotional component of life events, that is, their pleasantness or unpleasantness, is related to other characteristics of the events and to mental health. This emotional facet of the experience is conceptualized as a distinct and separate element from the cognitive evaluation of the desirability of life events, and perhaps the prime indicator of their effect on mental health.

\section{A MODEL FOR LINKING THE CONTROL, ANTICIPATION, STRESS, AND UNPLEASANTNESS OF LIFE EVENTS WITH WELL-BEING}

From the above-cited literature and concepts we have derived the model displayed in Figure 1. The model can be described by the following set of unfolding hypotheses.

1. Mental health or well-being should increase effective functioning. Functioning refers to an evaluation of how well the person manages his or her life roles and emotions. For example, how well does the person get along with others?

2. Mental health, in turn, is hypothesized to be reduced by the stress produced by events and increased by adjustment to these events. There is a considerable literature documenting the undesirable effects of a variety of stressors on mental health and on various emotional states in settings rang-

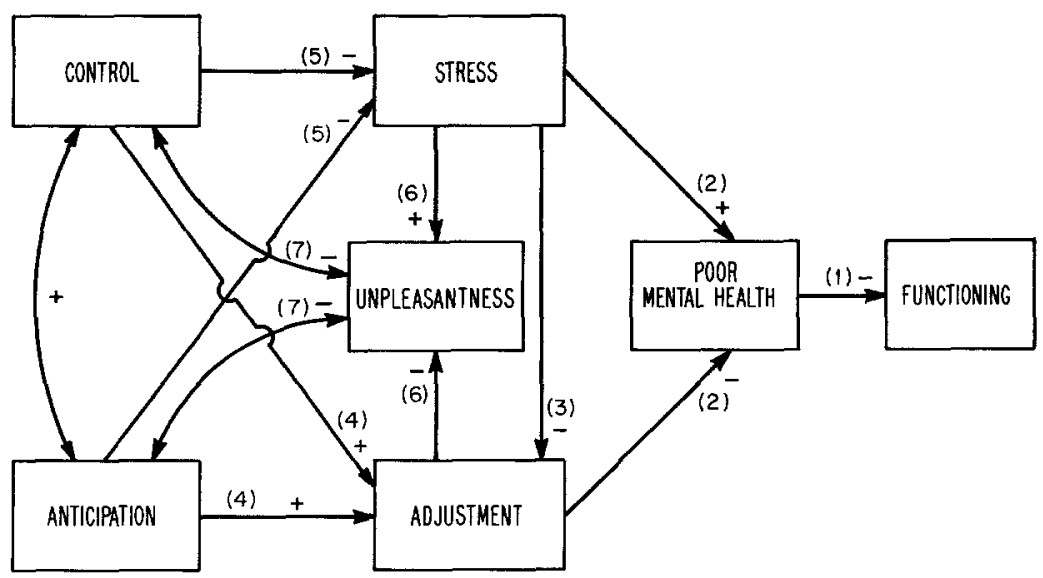

Fig. 1. Hypothesized effects of control, anticipation, stress, and adjustment on mental health and functioning. The arrows represent causal paths, with the plus and minus signs referring to hypothesized positive or negative relations, respectively. The numbers above the arrows refer to the hypothesis number in the text. 
ing from work (e.g., French, Caplan, \& Harrison, 1982; House, 1981) to school (e.g., Kulka, Klingel, \& Mann, 1980), and from among the elderly (Kahana, Liang, \& Felton, 1980) to the family (e.g., Gurin, Veroff, \& Feld, 1960). Adjustment is seen as promotive of well-being because the mere act of mastery as well as its outcomes may lead to enhancement of the self (DeCharms, 1968).

3. The stress of life events (independent of the mere occurrence of such events) should decrease the likelihood of making good adjustment: the higher the stress, the more difficult it is to adjust to it, and consequently, the lower the level of adjustment.

4. Both the anticipation of and the control over life events should improve the adjustment.

5. Similarly, both anticipation of and control over life events should reduce the level of stress brought about by life events. The rationale for this hypothesis is the assumption that both control and anticipation provide means to prepare for, modify, or avoid some of the outcomes and their unpleasant aspects.

6. The degree of stress evoked by the event and, in part, the difficulty or inability to adjust to it should determine the experience of unpleasantness associated with a life event. Thus, events that produce a great deal of stress and are difficult to adjust to are particularly likely to produce unpleasant affect.

7. Finally, uncontrollability and unpredictability characterize unpleasant life events insofar as most uncontrollable and unpredictable events are unpleasant. No causal influence is implied, however, because certain uncontrollable and unpredictable events can be just as pleasant as controllable and predictable events (e.g., winning a lottery).

In sum, this model's system of hypotheses attempts to integrate (a) cognitive aspects of events such as controllability and predictability, (b) affective aspects of events, such as degree of unpleasantness, and (c) the resultant stress and adjustment with the person's emotional well-being and functioning. This study represents one of the first attempts to empirically explore all of these links and evaluate their relative contributions.

\section{Subjective and Objective Measurements of Constructs in the Model}

It is often preferable to obtain objective measures that are not based on the respondent's perceptions (Kasl, 1984). Nevertheless, when it comes to measuring the concepts of predictability, control, and stress, the objective measures may not be the critical ones. Thompson (1981) has pointed out that even in the study of an objective stressor such as pain "It has long been noted... that the meaning of an injury or its consequences for the in- 
dividual dramatically affect the amount of pain felt (Beecher, 1956; Melzack \& Wall, 1965)." Similarly, Averill (1973) pointed out that the relationship of personal control to stress is primarily a function of the meaning of the control response for the individual.

This study focused on the meaning of the events to those who experience them; and consequently, we have obtained the respondents' own subjective assessment of their various experiences. As will later be described, the assessments of the life events and their related aspects was performed on a separate occasion and prior to the assessment of mental health. This collection procedure should minimize response contamination between the two types of measures. Furthermore, independent assessments of the respondents' role and emotional functioning were obtained from their significant others (e.g., spouse, close friends).

\section{METHODS}

\section{Respondents}

The respondents included 440 of 486 males from a third-wave followup of a longitudinal study which began 15 months earlier. The orginal group of respondents was drawn from a population living in Detroit and in its outlying areas. It included respondents who were predominantly white $88 \%$ white, $12 \%$ black), between 24 to 41 years of age (with mean age of 32), and most of them married $(71 \%)$. These respondents were drawn to represent employed and unemployed Vietnam veterans, era veterans (in the military during the Vietnam war but stationed elsewhere), and nonveterans of the same generation. They all volunteered to participate in the study and signed an informed consent form. The 297 unemployed respondents were recruited from nine state unemployment offices in southeast Michigan. The 189 employed respondents were nominated by their unemployed counterparts to intentionally produce demographically matched groups. This intent was met successfully (Vinokur, Caplan, \& Williams, in press). The recruitment procedure yielded a response rate of .84 and .86 for the employed and unemployed samples, respectively. The sampling design was intended to serve the purpose of the orgininal larger study (Vinokur et al., in press) and does not concern the present investigation.

\footnotetext{
${ }^{4}$ The work reported below is based on a larger research investigation on the effects of unemployment and participation in the Vietnam war on mental health and adjustment. A more comprehensive and detailed description of the methods and the measures is available from the authors.
} 


\section{Procedure}

With the exception of the data on life events, the data on all other topics were collected from the respondents through personal interviews. The life events data were collected using a self-administered questionnaire which was mailed to the respondents 2 weeks prior to the interview, with instructions on how to fill it out. In about $20 \%$ of the cases the interviewer had to provide additional explanation on how to fill out the forms of the life event questionnaire before the interview began. Of the 442 respondents who were interviewed, 420 completed successfully the life event questionnaire.

The personal interviews took place at the respondent's location of choice, usually his home, and they lasted for about 1 hour. Upon completion of the interview, the respondent was paid $\$ 5$ for his participation.

Each respondent selected a significant other (SO) who was asked to complete a self-administered questionnaire that provided another perspective on the respondent's adjustment or performance, mental health, and well-being. The significant other was someone who knew the respondent well and who saw the respondent at least once a week. Most of the significant others were the wives of the respondents (about $71 \%$ ) or close friends and girl friends (about 16\%).

\section{Questionnaire Instruments and Measures}

To distinguish measures from constructs, the names of the measures are capitalized in the text (e.g., Stress, Adjustment), whereas the names of the constructs are not (e.g., stress, adjustment).

Life Event Questionnaire. The questionnaire included 65 life events. The list of events was based on the original Holmes and Rahe (1967) instrument and included a number of additional events, which largely were more specific events than the ones in the original instrument. The respondents were asked to check the events that happened to them during the 14- to 16-month period between January 1, 1982 and the time of the interview, February to April of 1983. For each of the events that they checked, the respondents were also instructed to provide ratings to the following five questions:

1. Was the event pleasant or unpleasant?

2. How much were you responsible for it happening?

3. How much did you anticipate it happening?

4. How much pressure and stress did you experience from it?

5. How much were you able to adjust to it?

The phrasing of the questions was intended to operationalize the concepts discussed above. For example, the first question was intended to assess 
the affective experience associated with the event as distinguished from the cognitive evaluation of the desirability of the event's outcome. The second question was phrased to capture the respondent's perceived control over the occurrence of the event in terms of responsibility for it happening.

The response scale for the first question included only the categories pleasant (coded 1) and unpleasant (coded 2). (In about $6 \%$ of the cases the response to this question was either left unanswered or the respondent indicated it was both pleasant and unpleasant.) The response scale for the last four questions used the following five categories: 1 . not at all; 2 . just a little; 3 . some; 4 . pretty much; 5 . a great deal.

To test hypotheses about the effects of the frequency of life events as compared to other hypothesized aspects of their experience, we constructed the following four types of indices:

1. The number of events that were checked.

2. The sum of the ratings across events that were given to each of the latter four questions mentioned above.

3. The mean rating. The mean rating was computed by dividing the sum of the ratings for an index by the corresponding number of events that were checked.

4. The unpleasantness ratio index. This ratio was constructed by subtracting the number of pleasant events from the number of the unpleasant ones, and dividing by the total number of events checked. The ratio ranges from -1 , when only pleasant events were checked, to +1 , when only unpleasant ones were checked.

Mental Health and Well-Being. An index of Poor Mental Health (PMH), so named because most of the items focus on negative affective states, was used to measure mental health symptomatology. The index was composed of the following subscales: anxiety, depression, resentment, low selfesteem, and low life satisfaction. The emotional states of anxiety and depression were based largely on the Hopkins Symptom Checklist (Derogatis, Lipman, Rickels, Unlenhuth, \& Covi, 1974). The measures of self-esteem and of life satisfaction were based on Rosenberg's (1965) self-esteem scale and on Andrew and Withey's (1976) global life satisfaction scale. The resentment subscale was based on a measure developed by Andrews and Blumenthal (1973). The alpha coefficients for the above-mentioned subscales ranged from .82 to .87 . These subscales were highly intercorrelated, and thus the five subscales were averaged to produce an overall Poor Mental Health index with a coefficient alpha of .84 .

Role and Emotional Functioning. The measure of role and emotional functioning was taken from Caplan et al. (1984). This 13-item index measure, with a reliability alpha coefficient of .94 , provided an assessment by the 
significant other of how well the respondent handled his roles, interpersonal relationships, and emotions. The measure included questions such as: "How well has he [the respondent] done in handling his responsibilities and daily demands?... getting along with others?... acting in a relaxed manner?... staying level headed?" etc.

\section{RESULTS AND DISCUSSION}

\section{Zero-Order Correlations Among Life Events, Functioning, and Mental Health}

The first analyses were intended to replicate the most common findings regarding the relations between life events and their various characteristics (e.g., anticipation, control, pleasantness) to mental health. This replication indicates the validity of the measures in comparison to previous studies.

To examine these relations, product-moment correlations were computed between the indices based on the total number of events checked, the ratio of unpleasantness index, the sum ratings of stress, control, anticipation, and adjustment, and the two dependent measures: poor mental health $(\mathrm{PMH})$ and role and emotional functioning. Additionally, in order to examine the question of whether the effects of life events result primarily from an accumulation of events or from some general level of stress independent of the accumulation of events, product-moment correlations between the indices based on mean rating of the dependent variables were also computed. The correlations for these two analyses are displayed in Table I.

Table $I$ is organized so that the total number of all events and ratings of those events (stress, control, anticipation, and adjustment) are presented first, followed by these same parameters for the pleasant events and then for the unpleasant events. Only statistically significant results are discussed, unless noted otherwise.

With only two exceptions, all the indices that were positively and significantly associated with PMH were negatively and significantly associated with good Functioning. Consistent with research cited earlier, the pleasant or unpleasant quality of the event was the most important determinant of its effect on mental health and functioning.

Number of Pleasant and Unpleasant Events. The correlation between the ratio of Unpleasant Events Index and PMH was .46 $(p<.001)$. The total number of unpleasant events had a stronger effect than the total number of pleasant events on PMH $(r=.47$ compared to $r=-.23$, both $p<.001)$. The negative correlation between pleasant events and $\mathrm{PMH}$ is an exception 
Table I. Product-Moment Correlations of Life Event Indices with Poor Mental Health $(\mathrm{PMH})$ and Reported Functioning $(n=420)$

\begin{tabular}{|c|c|c|c|c|}
\hline \multirow{2}{*}{$\begin{array}{l}\text { Indices of } \\
\text { life events }\end{array}$} & \multicolumn{2}{|c|}{$\begin{array}{c}\text { Correlations } \\
\text { based on sum ratings }{ }^{a} \\
\end{array}$} & \multicolumn{2}{|c|}{$\begin{array}{c}\text { Correlations } \\
\text { based on mean ratings } \\
\end{array}$} \\
\hline & PMH & Functioning & PMH & Functioning \\
\hline $\begin{array}{l}\text { Total no. of all events } \\
\text { Ratio of unpleasant events } \\
\text { Stress } \\
\text { Control } \\
\text { Anticipation } \\
\text { Adjustment }\end{array}$ & $\begin{array}{l}.21^{f} \\
.46^{f} \\
.44^{f} \\
.05 \\
.05 \\
.00\end{array}$ & $\begin{array}{l}-.04 \\
-.19^{f} \\
-.15^{e} \\
.03 \\
.05 \\
.07\end{array}$ & $\begin{array}{r}- \\
.46^{f} \\
.54^{f} \\
-.26^{f} \\
-.29^{f} \\
-.50^{f}\end{array}$ & $\begin{array}{c}- \\
-.19^{f} \\
-.25^{f} \\
.12^{d} \\
.16^{e} \\
.21^{f}\end{array}$ \\
\hline $\begin{array}{l}\text { Pleasant events } \\
\text { Total no. of pleasant } \\
\text { events } \\
\text { Stress } \\
\text { Control } \\
\text { Anticipation } \\
\text { Adjustment }\end{array}$ & $\begin{array}{l}-.23^{f} \\
.02 \\
-.27^{f} \\
-.29^{f} \\
-.29^{f}\end{array}$ & $\begin{array}{l}.10^{d} \\
.00 \\
.15^{e} \\
.18^{f} \\
.15^{e}\end{array}$ & $\begin{array}{r}- \\
.40^{f} \\
-.19^{f} \\
-.17^{e} \\
-.31^{f}\end{array}$ & $\begin{array}{r}- \\
-.14^{d} \\
.14^{e} \\
.15^{e} \\
.16^{f}\end{array}$ \\
\hline $\begin{array}{l}\text { Unpleasant events } \\
\text { Total no. of unpleasant } \\
\text { events } \\
\text { Stress } \\
\text { Control } \\
\text { Anticipation } \\
\text { Adjustment }\end{array}$ & $\begin{array}{l}.47^{f} \\
.51^{f} \\
.40^{f} \\
.42^{f} \\
.31^{f}\end{array}$ & $\begin{array}{l}-.16^{e} \\
-.16^{e} \\
-.13^{e} \\
-.12^{d} \\
-.04\end{array}$ & $\begin{array}{c}- \\
.38^{f} \\
.12^{d} \\
.04 \\
-.40^{f}\end{array}$ & $\begin{array}{c}- \\
-.22^{f} \\
-.10^{d} \\
.00 \\
.22^{f}\end{array}$ \\
\hline
\end{tabular}

${ }^{a}$ Sum of ratings for stress, control, anticipation, or adjustment to the life events that were checked.

${ }^{b}$ The sum indices (see footnote $a$ ) divided by the respective number of events checked.

${ }^{c}$ This ratio ranges from -1 when all events are pleasant, to 0 when number of pleasant events equals number of unpleasant events, to +1 when all the events are unpleasant.

${ }^{d} p<.05$.

${ }^{e} p<.01$.

${ }^{f} p<.001$.

to previous research, which generally has failed to find any ameliorative effect of desirable events.

Controlling for Number of Events. In order to examine the possible effects of control, anticipation, stress, and adjustment on mental health and functioning, unconfounded by the number of events, we examined the correlations between the indices based on the mean ratings, and the dependent variables (see last two columns of Table I). For both pleasant and unpleasant events, mean level of Stress was correlated positively with Adjustment and negatively with PMH. For pleasant events, the correlations of Stress and Adjustment with PMH were .40 and -.31 , respectively (both $p<.001$ ). For the unpleasant events these correlations were .38 and -.40 (both $p<$ $.001)$, respectively. There was a similar effect of Stress on PMH and on Functioning regardless of whether the stress was from unpleasant or pleasant events. 
For pleasant events, the more respondents reported being able to control and anticipate such events, the lower was their PMH score $(r=-.19$ and -.17 , respectively; $p<.01$ ). For the unpleasant events, however, Control had a very weak effect on PMH $(r=.12, p<.05)$, and Anticipation had no effect.

Relations Among Qualitative Aspects of Events. Table II presents the intercorrelations among all the qualitative aspects of the events. Unpleasantness produced the most consistent pattern of strong associations with all the rest of the variables, and the $r s$ ranged from .50 to .59. As expected, Control and Anticipation were highly intercorrelated $(r=.57, p<.001)$ as were the correlations between Adjustment and Control, and between Adjustment and Anticipation $(r=.51$ and .52 , respectively, both $p<.001)$. The correlations between Stress and Control and between Stress and Anticipation, were relatively weak but statistically isignificant $(r=-.14$ and $-.27, p<$ .01) suggesting they are conceptually independent facets of life events.

\section{Multivariate Analyses}

This next series of analyses tested the hypotheses regarding the direct and indirect influence of the preceding variables on mental health and functioning. A series of multiple regression path analyses were performed using the indices that consisted of the mean ratings of Control, Anticipation, Stress, and Adjustment for the respondents. Multiple regressions were computed regressing all the variables first on the Functioning index, and second (excluding the functioning index) on PMH. Then, regressions were performed on adjustment using Stress, Control, and Anticipation as independent variables, and on Stress using Control and Anticipation as independent variables. The fifth analysis included a regression on the Unpleasantness Ratio Index with Stress and Adjustment as the independent variables. Figure 2 displays the results of these analyses, which consist of the significant paths, the standardized regression coefficients, and the multiple correlations.

Table II. Product-Moment Correlations Among Stress-Related Dimensions of Life Events ( $n=420$ Respondents)

\begin{tabular}{lrrrrr}
\hline \multicolumn{1}{c}{ Index measure } & 1 & 2 & 3 & 4 & 5 \\
\hline 1. Control & - & & & & \\
2. Anticipation & $.57^{b}$ & - & & & \\
3. Unpleasantness & $-.54^{b}$ & $-.50^{b}$ & - & & \\
4. Stress & $-.14^{a}$ & $-.27^{b}$ & $.59^{b}$ & - & \\
5. Adjustment & $.51^{b}$ & $.52^{b}$ & $-.57^{b}$ & $-.40^{b}$ & - \\
\hline${ }^{a} p<.01$. & & & & & \\
${ }^{b} p<.001$. & & & & & \\
\end{tabular}




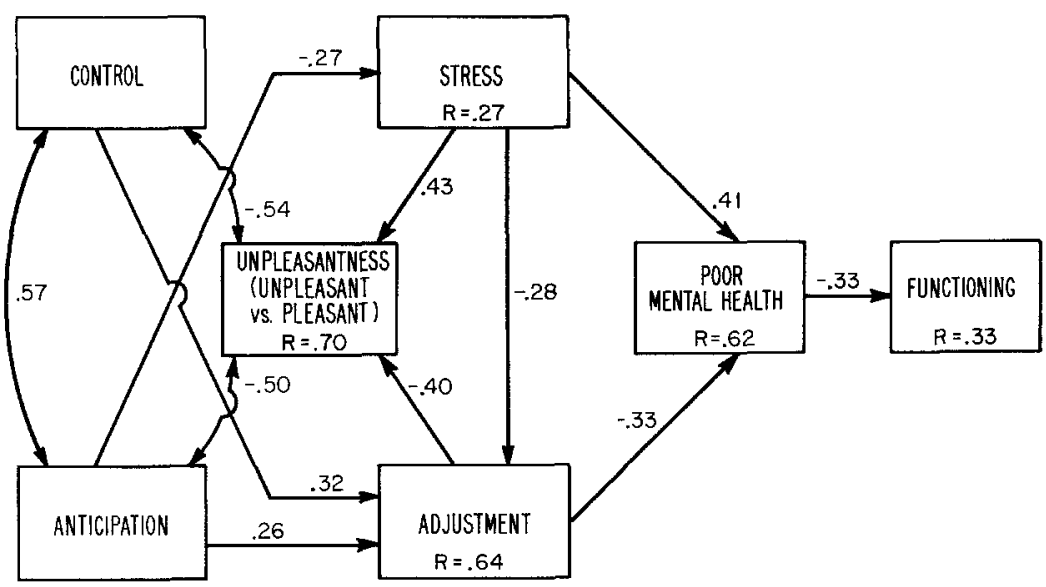

Fig. 2. Path diagram of the effects of life events experiences on poor mental health and functioning. Only statistically significant paths beyond the .05 level are presented with their coefficients (i.e., standardized regression beta weights). The multiple correlation coefficients $(R)$ are statistically significant at the .001 level.

As hypothesized, Functioning was adversely affected by Poor Mental Health $(\beta=-.33, p<.001)$. In turn, Mental Health appeared to be direct$1 \mathrm{y}$ affected by Stress and Adjustment. Whereas Stress and Adjustment had direct but opposite effects on PMH $(\beta=.41,-.33$, respectively, both $p$ $<.001$ ), neither Control nor Anticipation had such a direct effect. Instead, Control appeared to have a direct positive influence on Adjustment $(\beta=$ $.32, p<.001$ ), and thus influenced PMH indirectly. Similarly, and as hypothesized, Anticipation had no direct effect on Mental Health but was found to be associated with better Adjustment $(\beta=.26, p<.001)$ and lower Stress $(\beta=-.27, p<.001)$.

Again as hypothesized, Unpleasantness was jointly determined by the level of Stress $(\beta=.43, p<.001)$ and by poor Adjustment $(\beta=-.40$, $p<.001)$. And as seen before, Unpleasantness was correlated with lack of Control and low Anticipation $(r=-.57$, and -.50 , respectively, both $p$ $<.001)$. In sum, what characterized the unpleasant events was low control over the events and low anticipation of their occurrence.

\section{Effects for Pleasant Compared to Unpleasant Events}

The next analyses examined the question of whether the relations among the variables in Figure 2 were the same or different for pleasant and for unpleasant events. The multiple regressions described above were performed again but separately for the pleasant and unpleasant events. The 


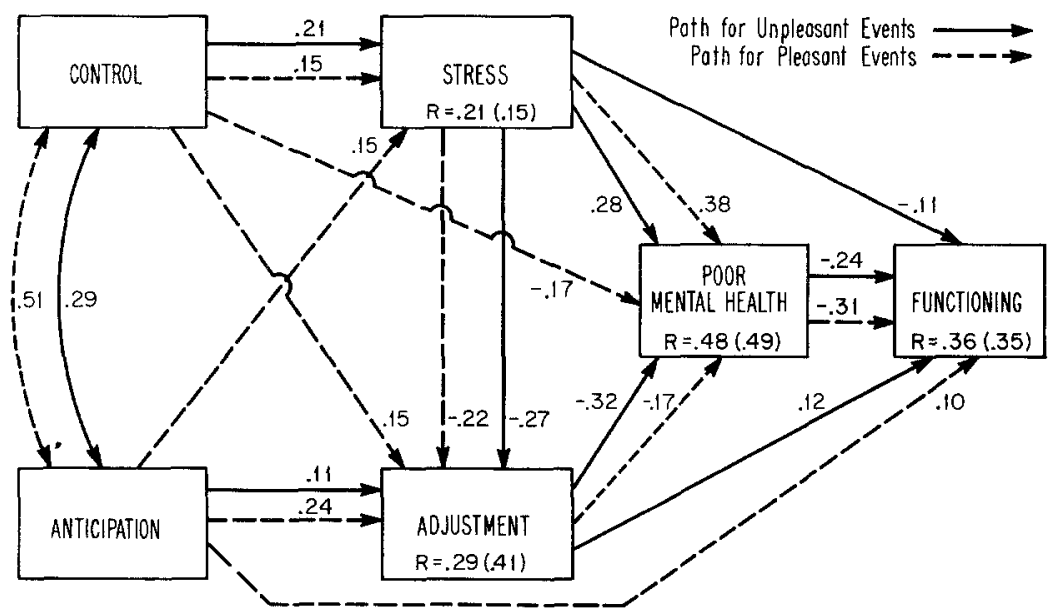

Fig. 3. Comparison of path diagrams for unpleasant and pleasant life events. The path diagram for the unpleasant events is represented by the solid arrows and the first multiple regression coefficient in the boxes. The path diagram for the pleasant events is represented by the dashed arrows and the second multiple regression coefficient in the boxes. All the path coefficients are standardized regression weights statistically significant beyond the .05 level. $R=$ multiple correlation coefficients for the unpleasant and pleasant events, respectively, all significant beyond the .001 level.

results are presented in Figure 3, with the solid lines and the dashed lines representing the paths for the unpleasant and the pleasant events, respectively. Where relevant, the multiple correlations coefficients for the unpleasant events appear first followed by the coefficient for the pleasant event.

Similarities in the Results for Pleasant and Unpleasant Events. For both unpleasant and pleasant events, PMH was predicted by Stress $(\beta=.28$ and .38 , respectively, both $p<.001)$, and by Adjustment $(\beta=-.32$ and -.17 , respectively, $p<.001)$. For both types of events, it appeared that the degree of Adjustment was positively affected by Anticipation $(\beta=.11, p<.05$, and $.24, p<.001$, respectively) and negatively affected by Stress $(\beta=-.27$ and -.22 , respectively, both $p<.001$ ).

Suppressor Effects. Contrary to our hypothesis (see hypothesis 5), control over the occurrence of the event seemed to result in an increase rather than a decrease in stress for both pleasant and unpleasant events $(\beta=.21$, $p<.001$, and .15, $p<.01$, respectively). It appears that the zero-order negative correlation between Control and Stress, as shown in Table II, was due largely to the negative relation between Unpleasantness and Control and the positive relation between Unpleasantness and Stress. The pattern of correlations in Table II suggests that the positive relation between Control and Stress was suppressed by the effect of Unpleasantness. Eliminating the ef- 
fect of unpleasantness produced partial correlations between control and stress that were positive $(r=.27, p<.05$, across the events, and $.26, p<.001$, across respondents). We examine the meaning of this finding in the Discussion.

Dissimilarities. Although Control, Anticipation, Stress, and Adjustment associated with pleasant or unpleasant events had in general similar effects on well-being, there were some differences. For pleasant events, control appeared to have direct ameliorative influences on PMH $(\beta=-.17, p<.01)$ and on Adjustment $(\beta=.15, p<.05)$. For unpleasant events there seemed to be only indirect effects. For pleasant events, Anticipation appeared to have direct effects on the reduction of Stress $(\beta=-.15, p<.05)$ and on improved Functioning $(\beta=.10, p<.05)$. For unpleasant events these effects were indirect. And finally, for unpleasant events, it appeared that Stress have a direct adverse effect, and Adjustment a direct beneficial effect, on Functioning $(\beta=-.11, .12$, respectively, both $p<.05)$. For the pleasant events these effects were only indirect.

\section{Control: It Increases Adjustment; Why Does it also Increase Stress?}

Although previously cited studies suggest that control over the occurrence of life events is generally associated with reduced stress, it now appears that this association should be attributed to the coincidental facts that most pleasant events are controllable and also evoke low stress and that most unpleasant events are uncontrollable and evoke high stress. When the degree of pleasantness of events was held constant statistically, control (over the occurrence of unpleasant as well as pleasant life events) heightened the stress evoked by the events. This suggests that even within pleasant events control over the occurrence of the events increases the stress associated with their experience. Greater control over the occurrence of events is possibly associated with greater stress regarding the decisions that produced them and also with the ensuing responsibilities for their outcomes (e.g., Bazerman, 1982; Rodin, Rennert, \& Solomon, 1980).

\section{How Do Multiple Events Influence Well-Being?}

The results of the analyses presented in Figures 2 and 3 were based on the mean ratings of the events rather than the sum of these ratings, and consequently, any effect of accumulation of change per se was effectively controlled for and eliminated. Similarly, the findings in the last two columns of Table I represent the effects of the average level of the various aspects of the life events and do not represent the accumulation of these aspects. 
If life events have a cumulative effect on perceptions of stress, and on adjustment, then the more events a person reports, the higher should be the person's average ratings of the stress produced by the events and the lower the adjustment. A cumulative effect might occur if previous exposure to events erodes the person's coping resources and the person's emotional resilience. To search for such an effect, we examined the extent to which the level of stress and adjustment was related to the sheer number of events that were experienced. Product-moment correlations were then computed between the indices based on mean ratings and on the number of events on which each index was based.

The correlations between the number of events checked by the respondent and the mean levels of Stress and Adjustment were $.28(p<.001)$ and $-.13(p<.01)$, respectively. For the pleasant events these correlations were $.02(\mathrm{~ns})$ and $.11(p<.05)$, respectively; for the unpleasant events they were $.33(p<.001)$ and $-.27(p<.001)$, respectively. Thus, these findings suggest that the accumulation of unpleasant events, but not of pleasant ones, increases stress and reduces adjustment. Possibly, each additional unpleasant event becomes more stressful than the previous ones.

\section{How Do Pleasant Events Influence Mental Health?}

Numerous investigations have shown that the effects of life events are primarily due to undesirable events (e.g., Thoits, 1983). This may be the case when all the events are analyzed together for the simple reason that desirable events produce relatively low level of stress. Our findings suggest that it is stress and adjustment, not the degree of pleasantness or unpleasantness of the event, that are the proximal determinants of mental health outcomes. As suggested by the data in Figure 3, even pleasant events can affect mental health adversely, but only to the extent that such events produce stress. As a whole, pleasant events produce lower levels of stress (mean $=1.97$ ) than unpleasant events (3.41), with a difference that is statistically significant ( $p$ $<.001$ ). They also facilitate better adjustment than unpleasant events (mean of 4.36 vs. 3.30 , respectively; difference $p<.001$ ). Consequently, pleasant events are least likely to have an adverse effect on mental health.

Another examination of the effects of pleasant life events on mental health focused on the net effect of the number of pleasant and unpleasant events on mental health. For this examination, multiple regression analyses were performed with the number of pleasant and unpleasant events as the independent variables and poor mental health and functioning indices as the dependent variables. In these analyses, the number of unpleasant and of pleasant events each had a statistically significant standardized beta weight. The 
beta weights for the number of pleasant and of unpleasant events for the poor mental health index were -.23 and .49 (both $p<.001$ ), respectively; and, for the role and emotional functioning index the betas were .10 and -.16 (both $p<.05$ ), respectively. The multiple $R$ s for the poor mental health and for the functioning indices were .54 and .19 (both significant at $p<$ .001 ), respectively. Although the unpleasant events contributed about twice as much to the variance of mental health as the pleasant events, the latter did have a significant, independent beneficial effect on mental health and well-being.

How does the finding that pleasant events promote emotional well-being relate to findings by others (e.g., Thoits, 1983) which show that desirable events have no effect on well-being? Earlier we pointed out that in previous studies on the desirability of events, respondents may have focused on and reported their cognitive evaluation of the events' outcomes. It is possible that the respondents, in assessing the pleasantness of the events in this study, focused more sharply on the emotional quality of the experience that accompanied the events. This suggests the hypothesis that emotional experience is a better predictor of the ultimate effects of life events on mental health than the cognitive evaluation of the events' outcomes. It is conceivable that there are many instances in which, when making an evaluation of desirability, people are not fully able to comprehend all the potential costs and benefits of an event (e.g., Nisbett \& Wilson, 1977). Under such conditions, any cognitive explanation of the event is likely to be unreliable (and hence, likely to have a weak effect on emotional well-being). In contrast, the affect that is generated in response to such events, regardless of whether clear cognitions are present or not, may be a more accurate indicator of the amount of stress produced by the event (Zajonc, 1980).

There are also the instances where the unpleasant experiences are followed by an outcome that is viewed as desirable (e.g., loss of a spouse after a painful terminal illness) and instances where pleasant experiences are followed by an undesirable outcome (e.g., a love affair followed by an unwanted pregnancy). It may be that the occurrence of such polar-opposite emotional experiences and outcomes accounts for the differences between the current findings and of those of earlier investigations. In earlier studies, respondents rated the desirability of events; in the present study they rated the pleasantness of events. It may be that the perceived pleasantness rather than the perceived desirability of events is a better indicator of the events effects on well-being. In order to formally test such hypotheses, future research needs to include assessments of desirability of the event's outcome as well as assessments of the experienced pleasantness of the event. 


\section{GENERAL DISCUSSION}

\section{Some Cautions in Interpretation}

The findings regarding the interrelations among characteristics of the life events (control, anticipation, stress, and adjustment) should be viewed with caution because they were collected from a single individual at a single sitting - a potential source of correlated measurement errors. Another, perhaps more serious, error may have resulted from a systematic cognitive or motivational bias. For example, to the extent that respondents were using some implicit theory (e.g., Eden \& Leviatan, 1975) of the effects of various aspects of life events on one another, the true relations among aspects of events might have been distorted.

Another caution relates to the cross-sectional and retrospective nature of the data collection. The path models suggest a plausible theory, but prospective designs are required to rule out competing explanations (e.g., poor mental health makes life events appear in retrospect more stressful and harder to adjust to than they were the time they occurred).

As noted earlier, we attempted to reduce some of the above pitfalls by collecting the data on life events several days prior to the collection of data on mental health and functioning. In addition, the data on functioning were obtained from the respondent's significant other rather than from the respondent himself. Our attempt did not guarantee the elimination of all the possible biases as we pointed out earlier. Nevertheless, the pattern of results, with only one exception regarding the effects of control on stress, supports the major hypotheses of the study.

\section{Implications for Future Research}

One value of the resulting model is its ability to show structurally how several key facets of life events are related to one another and how they influence well-being and role functioning. Such a model can serve as an anchor point to which one can link other theory and concepts relating to stress-coping processes.

For example, one can link social support theory to the model. A prolific amount of research on social support and the buffering hypothesis of social support (e.g., reviews by Thoits, 1982; Wortman, 1984) has focused largely on whether such an effect occurs and on the methodological issues that determine whether or not buffering is detected (e.g., Kessler \& McCleod, 1985). With rare exception (e.g., Caplan, 1979), there has been little at- 
tention paid to why buffering takes place. It is conceivable that social support may be directed towards and may influence any of the facets of life events that we have studied and any of the variables in the model that intervene between such events and well-being (such as the adjustment process). It is a logical next step to take the present model (Figures 2 and 3) and map in the role of facet-specific forms of social support aimed at control, anticipation, stress, adjustment, and so forth. Such research requires measures of social support whose content is generated on the basis of the theory presented in this and related studies of the facets of life events.

Lastly, although we are impressed with how much life events theory and knowledge has grown in complexity since its inception nearly 20 years ago, the theory has yet to reach its limit. For example, our model does not include several important facets of life events such as the stage of life when the event occurs, duration of the event (e.g., acute compared to chronic illness), and so on. If such facets go unmeasured and have important effects on well-being, our theories of life events will continue to fall significantly short of their potential as explanatory tools. One alternative may be to select and study a limited number of specific events of a particular type (e.g., loss events, Paykel et al., 1969, or, more specifically, loss of a spouse) so that one can control for the many unknowns that distinguish one phenotype of event from another. Such a research model may help us estimate the magnitude of impact that control, anticipation, stress, adjustment, and other facets of life events can have in determining how people cope with the specific adversities of life.

At present, no one approach addresses adequately the need for information about life events in general or about specific life events (e.g., job loss, marriage, and birth of a child). The study of comparable models and issues at both general and specific levels, however, could move our science towards a fully integrated view of life events.

\section{REFERENCES}

Andrews, F. M., \& Blumenthal, M. (1973). Justifying violence. Ann Arbor: Institute for Social Research.

Andrews, F. M., \& Withey, S. B. (1976). Social indicators of well-being: Americans' perceptions of life quality. New York: Plenum Press.

Averill, J. R. (1973). Personal control over aversive stimuli and its relationship to stress. Psychological Bulletin, 80, 286-303.

Bazerman, M. H. (1982). Impact of personal control on performance: Is added control always beneficial? Journal of Applied Psychology, 67, 472-479.

Beecher, H. K. (1956). Relationship of significance of wound to pain experienced. Journal of the American Medical Association, 161, 1609-1613. 
Caplan, R. D. (1979). Social support, person-environment fit, and coping. In L. Ferman \& J. Gordus (Eds.), Mental health and the economy (pp. 89-138). Kalamzoo MI: W. E. Upjohn Institute for Employment Research.

Caplan, R. D., Abbey, A., Abramis, D. J., Andrews, F. M., Conway, T. L., \& French, J. R. P. (1984). Tranquilizer use and well-being: A longitudinal study of social and psychological effects (Technical Report Series). Ann Arbor: University of Michigan, Institute for Social Research.

Cohen, S. (1980). After effects of stress on human performance and social behavior: A review of research and theory. Psychological Bulletin, 8, 82-108.

DeCharms, R. (1968). Personal causation: The internal affective determinants of behavior. New York: Academic Press.

Derogatis, L. R., Lipman, R. S., Rickels, K., Uhlenhuth, E. H., \& Covi, L. (1974). The Hopkins Symptoms Checklist (HSCL): A measure of primary symptom dimensions. Pharmacopsychiatry, 7, 79-110.

Eden, D., \& Leviatan, U. (1975). Implicit leadership theory as a determinant of the factor structure underlying supervisory behavior scales. Journal of Applied Psychology, 60, 736-741.

Folkman, S. (1984). Personal control and stress and coping processes: A theoretical analysis. Journal of Personality and Social Psychology, 46, 839-852.

Fontana, A. F., Hughes, L. A., Marcus, J. L., \& Dowds, B. N. (1979). Subjective evaluation of life events. Journal of Counseling and Clinical Psychology, 47, 906-911.

French, J. R. P., Jr., Caplan, R. D., \& Harrison, R. V. (1982). The mechanisms of job stress and strain. London: Wiley.

French, J. R. P., Jr., Rogers, W., \& Cobb, S. (1974). Adjustment as person-environment fit. In G. V. Coelho, D. A. Hamburg, \& J. E. Adams (Eds.), Coping and adaptation. New York: Basic Books.

Gersten, J. C., Langner, T. H., Eisenberg, J. C., \& Orzek, L. (1974). Child behavior and life events: Undesirable change or change per se? In B. S. Dohrenwend \& B. P. Dohrenwend (Eds.), Stressful life events: Their nature and effects. New York: Wiley.

Gurin, G. J., Veroff, \& Feld, S. C. (1960). Americans view their mental health. New York: Basic Books.

Holmes, T. H., \& Rahe, R. H. (1967). The social readjustment rating scale. Journal of Psychosomatic Medicine, 11, 213-218.

House, J. S. (1981). Work stress and social support. Reading, MA: Addison-Wesley.

Janis, I. L. (1962). Psychological effects of warning. In G. W. Baker \& D. W. Chapman (Eds.), Man and society in disaster. New York: Basic Books.

Kahana, E., Liang, J., \& Felton, B. J. (1980). Alternative models of person-environment fit: Prediction of morale in three homes for the aged. Journal of Gerontology, 35, 584-595.

Kasl, S. V. (1984). When to welcome a new measure. American Journal of Public Health, 74, 106-108.

Kessler, R. C., \& McLeod, J. D. (1985). Social support and mental health in community samples. In S. Cohen \& L. Syme (Eds.), Social support and health. (pp. 219-240). New York: Academic Press.

Kulka, R. A., Klingel, D. M., \& Mann, D. W. (1980). School crime and disruption as a function of student school fit: An empirical assessment. Journal of Youth and Adolescence, 9, 353-370.

McGrath, J. E. (1970). A conceptual formulation for research on stress. In J. E. McGrath (Ed.), Social and psychological factors in stress (pp. 10-21). New York: Holt, Rinehart \& Winston.

Melzack, R., \& Wall, P. D. (1965). Pain mechanisms: A theory. Science, 17, 287-304.

Mueller, D. P., Edwards, D. W., \& Yarvis, R. M. (1977). Stressful life events and psychiatric symptomology: Change or undesirability. Journal of Health and Social Behavior, 18, 307-317,

Nisbett, R. E., \& Wilson, T. D. (1977). Telling more than we can know: Verbal reports on mental processes. Psychological Review, 84, 231-259.

Paykel, E. S., Myers, J. K., Dienelt, M. N., Klerman, G. L., Lindenthal, J. J., \& Pepper, M. (1969). Life Events and depression: A controlled study. Archives of General Psychiatry, $21,753-760$. 
Rosenberg, M. (1965). Society and the adolescent self image. Princeton: Princeton University Press.

Rodin, J., Rennert, K., \& Solomon, S. K. (1980). Intrinsic motivation for control: Fact or fiction. In A. Baum \& J. E. Singer (Eds.) Advances in environmental Psychology: Applications of personal control (Vol. 2). Hillsdale, NJ: Lawrence Erlbaum.

Ross, C. E., \& Mirowsky, J., II. (1979). A comparison of life-event-weighting schemes: Change, undesirability, and effect-proportional indices. Journal of Health and Social Behavior, 20, 166-177.

Thoits, P. A. (1982). Life stress, social support, and psychological vulnerability: Epidemiological considerations. Journal of Community Psychology, 10, 341-362.

Thoits, P. A. (1983). Dimensions of life events that influence psychological distress: An evaluation and synthesis of the literature. In H. B. Kaplan (Ed.), Psychosocial stress. New York: Academic Press.

Thompson, S. C. (1981). Will it hurt less if I can control it? A complex answer to a simple question. Psychological Bulletin, 90, 89-101.

Wortman, C. B. (1984). Social support and cancer: Conceptual and theoretical issues. Cancer, 53, 2339-2360.

Vinokur, A., Caplan, R. D., \& Williams, C. C. (in press). Effects of recent and past stress on mental health: Coping with unemployment among Vietnam veterans and nonveterans. Journal of Applied Social Psychology.

Vinokur, A., \& Selzer, M. L. (1975). Desirable versus undesirable life events: Their relationship to stress and mental distress. Journal of Personality and Social Psychology, 32, 329-337.

Zajonc, R. B. (1980). Feeling and thinking: Preferences need no inferences. American Psychologist, 35, 151-175. 